\title{
Determining Health Utilities for Immunosuppression in Pediatric Inflammatory Bowel Disease
}

Lauren Schmidt, BA ${ }^{1}$, William E Bennett, Jr., MD, $\mathrm{MS}^{2}$

1. Indiana University School of Medicine, Indianapolis, IN 46202

2. Center for Pediatric and Adolescent Comparative Effectiveness Research, Department of Pediatrics, Indiana University School of Medicine, Indianapolis, IN 46202

3. Section of Pediatric Hematology and Oncology, Department of Pediatrics, Indiana University School of Medicine, Indianapolis, IN 46202

4. Section of Pediatric Gastroenterology, Hepatology, and Nutrition, Department of Pediatrics, Indiana University School of Medicine, Indianapolis, IN 4620

Background and Hypothesis: Inflammatory Bowel Disease is a significant cause of gastrointestinal pathology in children and adolescents, with increasing incidence. The medications used to control this disease have an overall low risk of detrimental side effects, but when they occur can be serious or lifethreatening. The most important side effects of immunosuppression in IBD are infection and malignancy, but little is known about how these risks affect patients' and their families' decisions about medication use. Clinical utilities are a standard methodology used to assign risk to various hypothetical health states. We hypothesized that lower health utility scores would be assigned to scenarios related to cancer than to infection, even if the risks or severity were similar. Experimental Design or Project Methods: This study determines the clinical utility of immunosuppression and cancer / infection risk in children with inflammatory bowel disease using the standard gamble technique. The standard gamble technique measures individual preferences for many different therapeutic modalities, under uncertain results. The study will look at the health utilities of at least 50 families at Riley hospital for Children and IU North, with at least 25 patients with ulcerative colitis and 25 patients with Crohn's disease. In addition to the perfect health and death, we propose to utilize six different hypothetical health states during this study: (1) untreated IBD, (2) on a medication with no side effects, (3) on a medication with risk of a treatable infection, (4) on a medication with risk of an untreatable infection, (5) on a medication with risk of a treatable cancer, (6) and on a medication with risk of an untreatable cancer. We will also gather subject demographics, family education, family income, and perform an assessment of numeracy. These variables will then be used in a regression model to assess the effect of these confounders.

Results: We have not yet enrolled subjects, but now that the tool and Standard Gamble design are complete, we expect to rapidly accrue enrollment over the next two weeks and will update results at that time.

Conclusion and Potential Impact: Health utility data in pediatric IBD are quite scarce, with room for further research. It is crucial to understand the health utilities in individuals with inflammatory bowel disease (IBD), specifically in pediatric patients. This allows for cost-utility analysis to play a role in determining effectiveness for a wide variety of treatment modalities. It is also important when discussing treatment options with families when the risk of infection or cancer are present. Knowledge gained from this study may help us design interventions that focus on educating families about the nature and magnitude of these important risks in immunosuppressive therapy. 